\title{
Cerebrospinal Fluid 7-Ketocholesterol Level Is Associated With Amyloid-ß42 and White Matter Microstructure in Cognitively Healthy Adults
}

Running head: Oxysterols, $A \beta_{42}$, and white matter microstructure

Ane Iriondo $^{\mathrm{a}}$, Maite García-Sebastian ${ }^{\mathrm{a}}$, Arantzazu Arrospide ${ }^{\mathrm{b}}$, Maria Arriba ${ }^{\mathrm{a}}$, Sara Aurtenetxe $^{\mathrm{a}}$, Myriam Barandiaran ${ }^{\mathrm{a}}$, Montserrat Clerigue ${ }^{\mathrm{a}}$, Mirian Ecay-Torres ${ }^{\mathrm{a}}$, Ainara Estanga $^{\mathrm{a}}$, Alazne Gabilondo ${ }^{\mathrm{a}}$, Andrea Izagirre ${ }^{\mathrm{a}}$, Jon Saldias ${ }^{\mathrm{a}}$, Mikel Tainta ${ }^{\mathrm{a}}$, Jorge Villanua $^{\mathrm{a}}$, Kaj Blennow ${ }^{\mathrm{c}, \mathrm{d}}$, Henrik Zetterberg ${ }^{\mathrm{c}, \mathrm{d}, \mathrm{e}, \mathrm{f}}$, Javier Mar ${ }^{\mathrm{b}}$, Beatriz Abad-García ${ }^{\mathrm{g}}$, Irundika HK Dias ${ }^{\text {h }}$, Felix M. Goñi ${ }^{i}$, Pablo Martínez-Lage ${ }^{\text {a }}$

${ }^{a}$ Center for Research and Advanced Therapies. CITA-Alzheimer Foundation. Mikeletegi 71, 20009 Donostia-San Sebastian, Gipuzkoa, Spain

${ }^{\mathrm{b}}$ Gipuzkoa Primary Care - Integrated Health Care Organizations Research Unit. Alto Deba Integrated Health Care Organisation, Nafarroa Hiribidea, 16, 20500 Arrasate, Gipuzkoa, Spain and Biodonostia Health Research Institute, Paseo Doctor Begiristain, s/n, 20014 Donostia - San Sebastian, Spain

${ }^{c}$ Department of Psychiatry and Neurochemistry, Institute of Neuroscience and Physiology, The Sahlgrenska Academy at University of Gothenburg, Mölndal, Sweden

${ }^{\mathrm{d}}$ Clinical Neurochemistry Laboratory, Sahlgrenska University Hospital, Mölndal, Sweden

${ }^{\mathrm{e}}$ Department of Neurodegenerative Disease, UCL Institute of Neurology, London, United Kingdom

${ }^{\mathrm{f}}$ UK Dementia Research Institute at UCL, London, United Kingdom

${ }^{g}$ Central Analysis Service, Faculty of Science and Technology, University of the Basque Country (UPV/EHU), Barrio Sarriena s/n, 48940 Leioa, Bizkaia, Spain

${ }^{\mathrm{h}}$ Aston Medical Research Institute, Aston Medical School, Aston University, Birmingham, B4 7ET, UK

${ }^{\text {i }}$ Departamento de Bioquímica, University of the Basque Country (UPV/EHU) and Instituto Biofisika (CSIC, UPV/EHU), Barrio Sarriena s/n, 48940 Leioa, Bizkaia, Spain

Corresponding Author:

Pablo Martinez-Lage

Fundación CITA-Alzheimer Fundazioa

Parque Científico tecnológico de Gipuzkoa, S.A.

Mikeletegi 71, bajo 20009 Donostia-San Sebastian (Gipuzkoa), Spain

Phone: 0034943.021 .792

pmlage@cita-alzheimer.org 
Title character count: 138

Running head character count: 49

Word count abstract: 250

Word count introduction: 404

Word count discussion: 1220

Word count manuscript:3829

Number of references: 50

Number of tables: 5

Number of figures: 2 


\section{ABSTRACT}

Background: Abnormal cholesterol metabolism changes the neuronal membrane and may promote amyloidogenesis. Oxysterols in cerebrospinal fluid (CSF) are related to Alzheimer's disease (AD) biomarkers in mild cognitive impairment and dementia. Cholesterol turnover is important for axonal and white matter (WM) microstructure maintenance.

Objective: We aim to demonstrate that the association of oxysterols, AD biomarkers, and WM microstructure occurs early in asymptomatic individuals.

Methods: We studied the association of inter-individual variability of CSF 24hydroxycholesterol (24-OHC), 27-hydroxycholesterol (27-OHC), 7-ketocholesterol (7$\mathrm{KC}), 7 \beta$-hydroxycholesterol (7 $\beta-\mathrm{OHC})$, amyloid- $\beta 42$ (A $\beta 42)$, total-tau (t-tau), phosphorylated-tau (p-tau), neurofilament (NfL), and WM microstructure using diffusion tensor imaging, generalized linear models and moderation/mediation analyses in 153 healthy adults.

Results: Higher 7-KC levels were related to lower A $\beta 42$, indicative of greater AD pathology $(\mathrm{p}=0.041)$. Higher 7 -KC levels were related to lower fractional anisotropy (FA) and higher mean (MD), axial (AxD), and radial (RD) diffusivity. 7-KC modulated the association between $\mathrm{AxD}$ and $\mathrm{NfL}$ in the corpus callosum splenium $(\mathrm{B}=39.39, \mathrm{p}=$ 0.017), genu ( $B=68.64, p=0.000)$, and fornix $(B=10.97, p=0.000)$. Lower $A \beta 42$ levels were associated to lower FA and higher MD, AxD, and RD in the fornix, corpus callosum, inferior longitudinal fasciculus, and hippocampus. The association between $\mathrm{AxD}$ and $\mathrm{A} \beta 42$ was moderated by $7 \mathrm{~K}-\mathrm{C}(\mathrm{p}=0.048)$. 
Conclusion: This study adds clinical evidence to support the role of $7 \mathrm{~K}-\mathrm{C}$ on axonal integrity and the involvement of cholesterol metabolism in the $\mathrm{A} \beta 42$ generation process. 


\section{INTRODUCTION}

Oxysterols have been proposed as the link between brain cholesterol metabolism and Alzheimer's disease (AD) ${ }^{1}$. In AD, oxidative stress enhances the formation of oxysterols, which in turn increase pro-inflammatory mediator production and exacerbate neuronal damage, connecting all these processes in a vicious circle ${ }^{2-4}$. Moreover, oxysterols play a fundamental role in APP-processing and $\beta$-amyloid - $_{1-42}\left(\mathrm{~A} \beta_{42}\right)$ generation as they induce changes in cell membrane dynamics ${ }^{5}$ that are related to the amyloidogenic processing in lipid rafts ${ }^{6,7}$. While experimental work suggests that oxysterols contribute to amyloidogenesis ${ }^{6-8}$, human studies have not shown an association between circulating oxysterols and CSF A $\beta_{42}$ levels ${ }^{9}$. Higher 24hydroxycholesterol (24OH-C) and 27-hidroxycholesterol (27OH-C), have been related to higher CSF total-tau (t-tau) and phosphorylated-tau (p-tau) levels in MCI and AD, but no in healthy people ${ }^{10,11}$.

To analyze the axolemma and myelin integrity, which are highly dependent on cholesterol metabolism and turnover, the assessment of white matter (WM) microstructure using DTI-MRI, is a valuable in vivo neuroimaging technique ${ }^{12}$. Tensorderived measurements include fractional anisotropy (FA) and radial, mean, and axial diffusivities (RD, MD, AxD) ${ }^{13}$. Despite the importance of cholesterol metabolism in the axolemma and myelin, few studies have analyzed the effect of cholesterol mismetabolism on the microstructure of $\mathrm{WM}^{14-16}$, and to our knowledge there is no previous work investigating the effect of oxysterols on WM microstructure. It is well established that patients with AD or mild cognitive impairment (MCI) show lower FA and higher MD, AxD and RD values especially in areas related to cognition, such as the

superior and inferior longitudinal fasciculus, hippocampus or corpus callosum ${ }^{17,18}$. Moreover other studies have shown WM microstructure changes in cognitively healthy 
people with positive amyloid biomarkers, especially in the fornix and the uncinate fascicle ${ }^{19,20}$.

To better understand the relationship between oxysterol homeostasis and neuronal integrity, we aimed to examine the association of CSF oxysterols, AD CSF biomarkers and WM microstructure, especially in regions associated to cognitive performance. For the present study we focus on the two major oxysterols synthetized enzymatically, 24hidroxycolesterol (24OH-C) and 27-hidroxycholesterol (27OH-C); and two synthetized non-enzymatically, $7 \beta$-hydroxycholesterol (7ßOH-C) and 7-ketocholesterol (7K-C). We hypothesized that inter-individual variability of CSF oxysterols is related to changes in DTI indexes and is associated to lower $A \beta_{42}$ and/or higher t-tau o p-tau levels. Furthermore, to investigate the role of inflammation and the occurrence of axonal damage, we have also looked at YKL-40 and neurofilament light (NfL).

\section{MATERIALS AND METHODS}

\section{Subjects}

This study is a cross-sectional analysis of baseline data from the Gipuzkoa Alzheimer Project (GAP). The GAP study is an ongoing longitudinal study on preclinical and prodromal phases of AD. A cohort of 410 non-demented volunteers (aged 40-80) was recruited from the community through local media advertisements and presentations at the local Alzheimer Association. Baseline visits took place between June 2011 and January 2013. Exclusion criteria were dementia and any significant neurologic, systemic, or psychiatric disorder causing cognitive impairment. All participants completed a comprehensive clinical and neuropsychological evaluation, anthropometric and cardiovascular risk assessment, blood workup, apolipoprotein E (APOE) genotyping for the $\varepsilon 2 / \varepsilon 3 / \varepsilon 4$ polymorphism, as well as brain magnetic resonance imaging 
(MRI). Lumbar puncture for CSF biomarkers was optional. For the present study was selected GAP participants deemed clinically normal based on a global Clinical Dementia Rating (CDR) score of $0^{21}$ with available CSF samples $(n=153)$. For the white matter microstructure analyses, 26 participants were excluded due to incidental findings and/or present of MRI artifacts identified by visual inspection and noted nonusable for this study.

The study was approved by the local Ethics and Clinical Research Committee, and all subjects gave written informed consent to participate.

\section{Magnetic Resonance Imaging (MRI)}

\section{$\underline{\text { Data acquisition }}$}

Whole brain scans were obtained using a Siemens 3T Magnetom TrioTim scanner (Siemens, Erlangen, Germany) in combination with a 32-channel head coil. To increase the inter-subject homogeneity of the image acquisition, the AutoAlign Head LS software tool (Siemens) was used. Diffusion-weighted images were obtained using an echo planar imaging (EPI) sequence with the following specifications: TR $9300 \mathrm{~ms}$, TE $92 \mathrm{~ms}$, voxel size: $1.7-\mathrm{mm}$ isotropic, 71 consecutive slices, acquisition matrix $122 \times 122$ (FOV $208 \mathrm{~mm}$ ), 6/8 partial Fourier, 64 diffusion directions with b-value $1000 \mathrm{~s} / \mathrm{mm} 2$, and one image with no diffusion weighting. The bandwidth was $1640 \mathrm{~Hz} /$ pixel. $\underline{\text { Image processing and analyses }}$

First, DICOM images were converted into 4D compressed NIfTI files and diffusion gradient directions were extracted with dcm2nii (part of MRIcron package, http://www.mccauslandcenter.sc.edu/mricro/mricron/). Then 4D brain volumes were processed and analysed by Tract-Based Spatial Statistics (TBSS), part of the FSL toolbox (FMRIB Software Library, Version 5.0.5; FMRIB, Oxford, United Kingdom) ${ }^{22-24}$. After image conversion, scalar DTI maps (FA, MD, AxD, and RD) were created 
by fitting a tensor model to the raw diffusion data using FDT (FMRIB's Diffusion Toolbox). Then FA images were brain-extracted using Brain Extraction Tool (BET) ${ }^{25}$, with $b=0$ as the reference volume. All subjects FA data were then aligned into a studyspecific FA template, using the nonlinear registration tool FNIRT ${ }^{26}$, which uses a bspline representation of the registration warp field ${ }^{27}$. The resulting warp-fields were then applied to MD, AxD and RD images. Due to the fact that in this study the subjects are middle to older age (40-77 years), we created a study-specific FA template instead of using the standard template (FMRIB58_FA) provided by FSL software, which is based on 58 subjects from 20 to 50 years. Before voxel-wise statistical analyses, all subject's FA data were obtained and projected onto a mean FA tract skeleton, which represents the centres of all tracts common to the group ${ }^{28}$.

\section{CSF biomarker measurements}

CSF was obtained and collected following international consensus recommendations as described previously ${ }^{29}$. Samples were aliquoted and stored in polypropylene tubes at $80^{\circ} \mathrm{C}$ and shipped on dry ice to the Clinical Neurochemistry Laboratory in Gothenburg for analysis. Biomarker concentrations were measured by board-certified laboratory technicians using commercial assays as previously described ${ }^{30}$ (MSD: $A \beta_{42}, A \beta_{40}$; Fujirebio Europe INNOTEST: t-tau and p-tau; R\&D: YKL-40; and UmanDiagnostics: NfL). As provided by the laboratory participants with $A \beta_{42} / A \beta_{40}<0.08 \mathrm{pg} / \mathrm{mL}$, were considered amyloid positive $(\mathrm{A} \beta+)$.

\section{Quantification of CSF oxysterols by LC-MS/MS}

Human CSF samples (400 $\mu \mathrm{l})$ spiked with internal standards (1 ng 24-OHCd7, $0.25 \mathrm{ng}$ 25-OHCd6, 4 ng 27-OHCd6, 0.5 ng 7 $\beta-O H C d 7,15$ ng 7-KCd7) was mixed with $1600 \mu 1$ ice-cold methanol containing $4 \mathrm{mg} / \mathrm{ml}$ butyl hydroxytoluene (BHT) and incubated for $10 \mathrm{~min}$ in ice. Samples were then centrifuged $\left(14,000 \mathrm{xg}\right.$ at $4^{\circ} \mathrm{C}$ for $\left.10 \mathrm{~min}\right)$ and the 
methanolic supernatant was diluted with acidified water up to $12.5 \%$ of methanol. Oxysterol enrichment and quantification was performed as described by Dias et al $2018^{31}$. Briefly, oxysterols were extracted using Oasis HLB Prime cartridges (bed wt. $30 \mathrm{mg}, 1 \mathrm{ml}, 96-$ well) and analysed by liquid chromatography UltiMate 3000 HPLC (Dionex, Thermo Scientific Ltd., Hemel Hempstead, UK) on-line coupled to the ESIQqLIT-MS/MS mass spectrometer (QTrap 5500, AB Sciex, Warrington, UK). Multiple reaction monitoring with transitions of 367.2/161 for 24-OHC, 367.4/ 147 for 25-OHC, 385.4/161 for $27-\mathrm{OHC}, 385.4 / 81$ for $7 \beta-\mathrm{OHC}$ and $401.4 / 95$ for $7-\mathrm{KC}$ were used to collect data. Data were examined using Analyst Software 1.7.2 (AB Sciex, Warrington, UK).

\section{APOE genotype}

APOE genotype was determined using 1-stage polymerase chain reaction as previously described ${ }^{32}$. Participants were classified as $A P O E \& 4$ carriers (APOE4+) if they had at least one $\varepsilon 4$ allele, or as non-carriers (APOE4-).

\section{White matter hyperintensities}

Individual global white matter hyperintensity (WMH) was semiquantified by the Fazekas scale by an experienced neuroradiologist on fluid attenuation inversion recovery MRI sequences. Fazekas scale ranges between 0 and $3(0=$ no $\mathrm{WMH} ; 1=$ focal/punctate lesions; 2 = beginning confluent lesions; 3 = confluent-diffuse lesions) ${ }^{33}$.

\section{Statistical Analysis}

To assess the appropriateness of parametric statistics, the Kolmogorov-Smirnov test was used to examine the normality of oxysterol distribution. Frequency distributions were calculated for categorical variables, and means (standard deviations) or median (percentile 25 -percentile 75 ) were calculated for continuous variables.

\section{$\underline{\text { Oxysterols and AD biomarkers }}$}


Separate generalized linear models (GLM) were conducted to explore the association between oxysterol measured by $24 \mathrm{OH}-\mathrm{C}, 27 \mathrm{OH}-\mathrm{C}, 7 \mathrm{~K}-\mathrm{C}, 7 \beta \mathrm{OH}-\mathrm{C}$ and $\mathrm{CSF}$ biomarkers (A $\beta_{42}$, t-tau, p-tau) adjusted by age, sex and $A P O E$ genotype. If a significant association was found, we examined the role of YKL40 including this variable in a new regression model.

\section{$\underline{\text { White matter microstructure }}$}

All DTI indexes, were correlated with age, sex and WMH, therefore all analysis were adjusted for these confounders. To test the association between oxysterols and WM microstructure as indexed by the DTI measurements (FA, MD, RD, AxD), whole-brain voxel-wise statistical analyses were performed with a nonparametric permutation inference tool (randomise, part of the FSL toolbox). For these analyses 5000 permutations were generated and Threshold-Free Cluster Enhancement (TFCE) was conducted as a thresholding option ${ }^{34}$. All the resulting statistical maps were corrected for multiple comparisons with family wise error (FWE), thresholded at $p<0.05$ and only clusters with at least 100 contiguous voxels were defined as significant clusters. Resulting significant clusters were labelled using the atlas available through FSL toolbox (ICBM-DTI-81 white matter labels atlas).

Based on previous results linking WM microstructure with cognitive performance ${ }^{17,35}$, special attention was focused on the following regions: corpus callosum, inferior and superior longitudinal fasciculus, cingulate gyrus, hippocampus, uncinate fasciculus, fornix (column+body) and fornix cres/stria terminalis These regions were labelled using the atlas available through FSL toolbox (ICBM-DTI-81 white matter labels atlas). If an oxysterol showed a significant association with an AD biomarker and a DTI index, the effect of the biomarker on the DTI index was analysed in the voxels where the oxysterol had shown a significant effect. Individual mean values for DTI indexes were 
extracted in regional clusters that were significantly associated with oxysterols according to TBSS analysis (FEW-corrected $p<0.05$ ). For these analyses, GLMs were conducted. The dependent variable was the mean of the DTI measures of each region and the predictor was the biomarker. The DTI indexes were multiplied by 1000 in the analyses ${ }^{36}$.

To better understand the association between oxysterols, biomarkers and WM microstructure in areas related to cognition, GLMs were designed for the DTI indexes obtained from each region where the oxysterol and biomarkers showed a significant effect. The biomarker was the dependent variable and oxysterol, DTI index and the interaction between both were predictive variables. Mediation and moderation analyses were performed to better understand the associations found between oxysterols, AD biomarkers and cognitive regions WM microstructure. Moderation is shown up by a significant interaction effect.

Finally, we conducted new GLMs to study the effect of the interaction between oxysterols and DTI indexes and between biomarkers and DTI indexes on CSF NfL levels, as a biomarker of axonal damage. Mediation and moderation analyses were performed to better understand the associations found between oxysterols, AD biomarkers, cognitive regions DTI indexes and NfL. All models were controlled by sex, age, WMH and $A P O E$ genotype.

Statistical analyses were conducted in SPSS version 20. For mediation and moderation analysis we applied the PROCESS macro for SPSS (version 3) by Andrew F.Hayes (http://www.afhayes.com).

\section{RESULTS}

Descriptive data for the whole sample $(\mathrm{n}=153, \mathrm{CDR}$ score $=0)$ are shown in Table 1. Participant age range was 40-75, 67 (43.8\%) were women and mean MMSE score was 29. 


\section{Oxysterols and CSF biomarkers for AD pathology}

To study the association between oxysterol levels and CSF AD biomarkers, GLMs were conducted among 153 healthy participants controlling for age, gender and APOE genotype. $7 \mathrm{~K}-\mathrm{C}$ was associated with $\mathrm{A} \beta_{42}(B=-0.20 ; p=0.041)$ (Table 2). There were no effects of $7 \mathrm{~K}-\mathrm{C}$ on t-tau or p-tau. The other oxysterols, $24 \mathrm{OH}-\mathrm{C}, 27 \mathrm{OH}-\mathrm{C}, 7 \beta \mathrm{OH}-\mathrm{C}$, did not show any significant effect on any CSF biomarker.

As $\mathrm{A} \beta_{42}$ was significantly correlated with $\mathrm{YKL} 40\left(r_{s}=0.244 ; p=0.003\right)$ and $7 \mathrm{~K}-\mathrm{C}$ is related to apoptosis and inflammation ${ }^{37}$ we introduced YKL40 in the model. YKL40 (B $=0.001 ; p=0.000)$ and $7 \mathrm{~K}-\mathrm{C}(B=-0.19 ; p=0.044)$ showed a significant effect on $\mathrm{A} \beta_{42}$.

. The interaction between YKL40 and 7K-C, was not significant $(B=-5.88 \mathrm{E}-7 ; p=$ $0.812)$.

\section{Oxysterols and white matter microstructure}

To study the correlation between oxysterols and WM microstructure, voxel-wise correlational analysis was conducted among 127 healthy participants controlling for age, gender and white matter hyperintensities. Descriptive data for the subsample with available MRI included significantly more women than the all sample $(p=0.005)$ (Table 1).

TBSS analysis detected a significant negative association between 7K-C and FA (Figure 1A). Most of these voxels were located in the corpus callosum. Significant associations were also seen in the following regions: left anterior and right posterior corona radiata, left external capsule, right hippocampus and right superior longitudinal fascicule. We did not observe significant positive association between 7K-C and FA. Analyses also revealed a significant positive association correlation between 7K-C and MD (Figure 1B), AxD (Figure 1C) and RD (Figure 1D) distributed throughout the whole brain WM in both hemispheres. We did not observe significant negative association between $7 \mathrm{~K}-\mathrm{C}$ 
and $\mathrm{MD}, \mathrm{AxD}$ or RD. Table 3 shows the distribution and proportion of voxels where a significant association between 7K-C and each DTI measure was detected in every cognitive region.

There was no significant effect for $24 \mathrm{OH}-\mathrm{C}, 27 \mathrm{OH}-\mathrm{C}$ and $7 \beta \mathrm{OH}-\mathrm{C}$ in any of the DTI measurements.

Oxysterols, CSF biomarkers for AD pathology and cognitive regions white matter microstructure

As $7 \mathrm{~K}-\mathrm{C}$ had a significant effect on $\mathrm{A} \beta_{42}$, the association between $\mathrm{A} \beta_{42}$ and DTI indexes of WM microstructure was analysed in the voxels where $7 \mathrm{~K}-\mathrm{C}$ showed a significant effect on each index (Table 3). GLMs were adjusted for age, gender, WMH and APOE genotype.

$\mathrm{A} \beta_{42}$ had a positive effect on FA of the splenium of the corpus callosum and AxD of right hippocampus, and a negative effect on the MD of the body of the corpus callosum, fornix (column+body) and left inferior longitudinal fasciculus; on $\mathrm{AxD}$ of the genu and splenium of the corpus callosum and fornix (Column+body); and on RD of the fornix (column+body) (Figure 2). The $\beta A+$ group $(n=25)$ had significantly higher $M D, A x D$ and $\mathrm{RD}$ values in the fornix (column+body) than $\beta \mathrm{A}_{-}$group $(\mathrm{n}=126)\left(\mathrm{MD}: \beta \mathrm{A}^{+}\right.$, median $=1.75 ; \beta \mathrm{A}-$, median $=1.42 ; p=0.001 / \mathrm{AxD}: \beta \mathrm{A}+$, median $=2.32 ; \beta \mathrm{A}-$, median $=2.03$; $p=0.003 / \mathrm{RD}: \beta \mathrm{A}^{+}$, median $=1.50 ; \beta \mathrm{A}-$, median $\left.=1.11 ; p=0.002\right)($ Figure 2$)$. To explore the association between $7 \mathrm{~K}-\mathrm{C}, \mathrm{A} \beta_{42}$ and $\mathrm{WM}$ microstructure, the effect of $7 \mathrm{~K}-\mathrm{C}$ and its interaction with the WM microstructure indexes $(\mathrm{FA} * 7 \mathrm{~K}-\mathrm{C}$; MD*7K-C; $\mathrm{AxD} * 7 \mathrm{~K}-\mathrm{C} ; \mathrm{RD} * 7 \mathrm{~K}-\mathrm{C})$ on the level of $\mathrm{A} \beta_{42}$ was studied in the areas related to cognitive performance where the $7 \mathrm{~K}-\mathrm{C}$ and $\mathrm{A} \beta_{42}$ showed significant effect. Higher MD, $\mathrm{AxD}$ and $\mathrm{RD}$ of the fornix, and higher $\mathrm{AxD}$ of the splenium and genu of the corpus callosum, right hippocampus and left inferior longitudinal fasciculus were related to 
lower $A \beta_{42}$ (Table 4). In these model 7K-C and the interactions between $7 \mathrm{~K}-\mathrm{C}$ and DTI measures were not significant. In the left inferior longitudinal fasciculus model, AxD, 7K-C and 7K-C*AxD interaction showed a significant effect. Moderation analyses showed that the relationship between $\mathrm{AxD}$ and $\mathrm{A} \beta_{42}$ was moderated by $7 \mathrm{~K}-\mathrm{C}$ level $(B=$ 3.87, $p=0.048)$. When YKL40 was included in the analyses, the moderation effect of $7 \mathrm{~K}-\mathrm{C}$ was not significant $(p=0.082)$.

\section{Oxysterols, CSF AD biomarkers, axial diffusivity and neurofilament,}

As $\mathrm{AxD}$ is related to axonal damage, the effect of the $7 \mathrm{~K}-\mathrm{C}^{*} \mathrm{AxD}$ interaction, and the $\mathrm{A} \beta_{42} * \mathrm{AxD}$ interaction on $\mathrm{NfL}$ for areas related to cognition where $7 \mathrm{~K}-\mathrm{C}$ and $\mathrm{A} \beta_{42}$ had significant effect were analyzed in separate GLMs. The 7K-C*AxD interaction showed a significant effect on NfL in the fornix and in the splenium and genu of the corpus callosum (Table 5). In these regions, the relationship between AxD and NfL was moderated by $7 \mathrm{~K}-\mathrm{C}$ (Fornix: $B=1.53, p=0.000$; splenium: $B=5.51, p=0.017$; Genu: $B=9.61, p=0.000)$. The $\mathrm{A} \beta_{42} * \mathrm{AxD}$ showed no significant effect on NfL in any region.

\section{DISCUSSION}

We have investigated the association between oxysterols, CSF AD biomarkers and WM microstructure in cognitive regions in a population of cognitively healthy middle-aged subjects. To our knowledge, this might be the first study on the effect of oxysterols on WM microstructure and the first to report that higher 7K-C levels are related to lower $\mathrm{A} \beta 42.7 \mathrm{~K}-\mathrm{C}$ had a negative effect on FA and a positive effect on $\mathrm{MD}, \mathrm{AxD}$ and $\mathrm{RD}$ in multiple cognitive regions. Moreover, $7 \mathrm{~K}-\mathrm{C}$ modulated the association between $\mathrm{AxD}$ and axonal loss, as measured by NfL, in the splenium and genu of the corpus callosum and fornix. Interestingly, in the voxels where $7 \mathrm{~K}-\mathrm{C}$ showed a significant effect on FA, $\mathrm{MD}, \mathrm{AxD}$ and $\mathrm{RD}, \mathrm{A} \beta_{42}$ also showed positive association with FA and negative effects 
on $\mathrm{MD}, \mathrm{AxD}$ and $\mathrm{RD}$ in the fornix, corpus callosum, inferior longitudinal fasciculus and hippocampus. Furthermore subjects who were $\beta A+$ had significantly higher $\mathrm{MD}, \mathrm{AxD}$ and $\mathrm{RD}$ values in the fornix than the $\beta \mathrm{A}$ - group.

Previous case-control studies on CSF oxysterol levels have reported that $24 \mathrm{OH}-\mathrm{C}$ and 27OH-C levels are higher in patients with $\mathrm{AD}$ or $\mathrm{MCI}{ }^{10,11,38}$. Other studies, analyzing patients with $\mathrm{AD}$ or MCI and controls al together, have shown positive correlations between $24 \mathrm{OH}-\mathrm{C}$ CSF concentrations and t-tau and p-tau levels, but not with $\mathrm{A} \beta_{42}{ }^{10,39}$. However these correlations were significant only in the AD or MCI group, and no in healthy people. Popp and colleagues described a positive correlation between $24 \mathrm{OH}-\mathrm{C}$ and sAPP $\alpha$ and sAPP $\beta$ in patients with AD, but no with $A \beta_{42}{ }^{40}$. Our results confirm the absence of association between $24 \mathrm{OH}-\mathrm{C}$ and $27 \mathrm{OH}-\mathrm{C}$ and CSF AD biomarkers in cognitively healthy adults. This suggests that if $24 \mathrm{OH}-\mathrm{C}$ and $27 \mathrm{OH}-\mathrm{C}$ are involved in AD pathophysiology this would not represent an early event. Although 24-OHC and 27$\mathrm{OHC}$ are the oxysterols most widely considered to be potentially implicated in $\mathrm{AD}$ pathogenesis, the possible involvement of oxysterols resulting from cholesterol autoxidation is now emerging. In fact, in a recent systematic analysis of oxysterols in post-mortem human AD brains, the level of some of the oxysterols deriving from cholesterol autoxidation were higher in subjects in the Braak I-II stages than in controls, and there were no differences in the levels of the two oxysterols of enzymatic origin ${ }^{41}$. $7 \mathrm{~K}-\mathrm{C}$ is a major product of reactive oxygen species (ROS)-caused oxidation of cholesterol ${ }^{42}$. We found that higher $7 \mathrm{~K}-\mathrm{C}$ levels were associated with lower $\mathrm{A} \beta_{42}$. This result is in line with previous model lipid membrane studies reporting a relation between $7 \mathrm{~K}-\mathrm{C}$ and $\mathrm{A} \beta_{42}{ }^{43,44}$. In the study by Kim and colleagues, they observed that partial substitution of cholesterol with $7 \mathrm{~K}-\mathrm{C}$ in the model lipid membrane enhances $\mathrm{A} \beta_{42}$ insertion into the lipid bilayer, by decreasing intramolecular cohesive interactions 
${ }^{44}$. In the same line, Phan and colleagues demonstrated that 7K-C renders lipid bilayer less condensed and more fluid than cholesterol, thus accelerating $A \beta_{42}$ association with the bilayer ${ }^{6}$, specially the protofibrillar $A \beta_{42}{ }^{43}$. Moreover, a recent model lipid membrane study demonstrated that cholesterol and 7K-C have different effects on membrane-mediated aggregation of $\mathrm{A} \beta_{42}$. While cholesterol inhibited the nucleation step and accelerated fibrillar $A \beta_{42}$ growth, the partial substitution of membrane cholesterol with 7K-C slightly increased the nucleation fase and remarkably decreased fibril elongation $^{45}$. The oligomers or protofibrillar $A \beta_{42}$ formed in the nucleation step are reportedly more toxic than soluble monomers and mature fibrils ${ }^{46}$. These papers suggested that cholesterol and $7 \mathrm{~K}-\mathrm{C}$ can modulate interaction of $\mathrm{A} \beta_{42}$ with cell membranes by influencing the fibrillation of the peptide. Our work adds clinical evidence to support these experimental studies on the relation between $7 \mathrm{~K}-\mathrm{C}$ and $\mathrm{A} \beta_{42}$. An in vitro study showed that 7K-C induced apoptosis in cells associated with increased ROS generation, ER stress and upregulated caspase-3/7 activity ${ }^{37}$. Thus, we hypothesized that neuroinflammation, as measured by YKL40 had an effect in the relation between $7 \mathrm{~K}-\mathrm{C}$ and $\mathrm{A} \beta_{42}$. Our results demonstrated that both, 7K-C and YKL40, have a significant effect on $\mathrm{A} \beta_{42}$, but there is no interaction between $7 \mathrm{~K}-\mathrm{C}$ and $\mathrm{YKL} 40$, suggesting that both influence the amyloidogenic process by different pathways. Regarding WM microstructure, to our knowledge, this might be the first study investigating the effect of oxysterols on WM microstructure. We found that $7 \mathrm{~K}-\mathrm{C}$ had a negative effect on FA and a positive effect on $\mathrm{MD}, \mathrm{AxD}$ and $\mathrm{RD}$ in multiple cognitive regions. Moreover, 7K-C modulates the relation between NfL, a marker of axonal damage $^{47}$, and AxD, the DTI index related to axonal damage ${ }^{48} .7 \mathrm{~K}-\mathrm{C}$ induces oxidative stress ${ }^{37}$ which in turn induces activation of microglia and astrocytes with a consequent increase of pro-inflammatory mediator production and exacerbating 
neuronal damage ${ }^{2}$. Our results suggest that the WM microstructure changes related to neuronal and axonal damage are related to altered cholesterol metabolism and increased membrane levels of $7 \mathrm{~K}-\mathrm{C}$. With respect to $\mathrm{A} \beta_{42}$, we found a relation between lower CSF $A \beta_{42}$ and lower FA and higher $\mathrm{MD}, \mathrm{AxD}$ or $\mathrm{RD}$ in areas related to cognitive performance such as fornix, corpus callosum, inferior longitudinal fasciculus and hippocampus in those voxels where $7 \mathrm{~K}-\mathrm{C}$ showed a significant effect on the DTI indexes. Previous studies have shown lower FA and higher MD in patients with AD, especially in areas such as corpus callosum, fornix, superior and inferior longitudinal fasciculus or cingulum ${ }^{17,18,49}$. Similar changes in WM microstructure are also found in cognitively healthy persons with abnormal $A \beta_{42}$ levels, specially in the uncinate fasciculus and fornix ${ }^{19,20}$. These findings are in line with our results. In our study $\beta A+$ subjects have significantly higher $\mathrm{MD}, \mathrm{AxD}$ and $\mathrm{RD}$ values in the fornix than the $\beta \mathrm{A}$ group.

We have found a relation between $\mathrm{A} \beta_{42}, 7 \mathrm{~K}-\mathrm{C}$ and $\mathrm{WM}$ microstructure in cognitive regions. Axon integrity depends mainly on the axolemma structure which in turn depends on cholesterol turnover. Our work would suggest that cholesterol metabolism and integration of $7 \mathrm{~K}-\mathrm{C}$ in the axolemma could change the structure of the membrane, alter WM microstructure and modulate the amyloidogenic process. The GAP study has allowed us to analyze a rigorously phenotyped cohort with strict clinical and cognitive criteria to exclude participants with cognitive impairment rigorously. However, the study must also be interpreted in the light of its limitations. The principal one is that DTI metrics are an indirect measure of WM microstructure and the influence of crossing fibers makes the interpretation more complicated in all DTI studies. However, the strength of the study is that participants performed a high resolution 3T-DWI with 65 directions which has allowed to realize robust estimates of 
tensor derived-properties ${ }^{50}$. This is a cross-sectional study, which cannot characterize how the association between $7 \mathrm{~K}-\mathrm{C}, \mathrm{A} \beta_{42}$ and $\mathrm{WM}$ microstructure changes over time. Longitudinal follow-up data on the GAP study cohort are now being collected and will be the subject of further analysis to confirm these cross-sectional findings. In conclusion, this study shows that interindividual variability of CSF 7K-C levels in healthy adults are associated with $A \beta_{42}$ levels and WM integrity in cognitive regions. Perhaps, for the first time, this study adds clinical evidence to support experimental studies on the potential effect of $7 \mathrm{~K}-\mathrm{C}$ in the $\mathrm{A} \beta_{42}$ aggregation process and membrane structure. Furthermore, this study reports, possibly for the first time, the effect of $7 \mathrm{~K}-\mathrm{C}$ on the WM microstructure and its modulating effect on the relation between axonal damage and white matter microstructure, suggesting that WM microstructure changes related to $7 \mathrm{~K}-\mathrm{C}$ could be a mirror of the neuronal damage induced by $7 \mathrm{~K}-\mathrm{C}$.

\section{Acknowledgments}

The authors gratefully acknowledge the volunteers (a Basque Cohort) who participate in the Gipuzkoa Alzheimer Project (GAP) for their generous collaboration. This work has been possible thanks to the support of the computing infrastructure of the i2BASQUE academic network (www-en.i2basque.es).

This work was supported by the Department of Economic Promotion, Rural Areas and Territorial Balance of the Provincial Government of Gipuzkoa (124/16); the Department of Health of the Basque Government (2016111096); and by the Carlos III Institute of Health (PI15/00919, PN de I+D+I 2013-2016). Predoctoral fellowship grant (Programa Predoctoral, de Formación de Personal Investigador no doctor, RBFI-2015-1-0231) was received from the Basque Government (AIJ). It was undertaken at CITA Alzheimer Foundation, Centre for Research and Advanced Therapies for Alzheimer's disease (http://www.cita-alzheimer.org/english.asp), which is supported by the Ministry 
of Economy and Competitiveness of Spain, the Basque Country Government, KutxaFundazioa and anonymous private sponsors. $\mathrm{HZ}$ is a Wallenberg Academy Fellow supported by grants from the Swedish Research Council (\#2018-02532), the European Research Council (\#681712) and Swedish State Support for Clinical Research (\#ALFGBG-720931).

\section{Author contributions}

AI and PML conception and design of the study; AI, MGS, MA, SA, MB, MC, MET, AE, AG, AIz., JS, MT, JV, KB, HZ, BAG, IHK, and PML acquisition of data; AI, AA, JM and PML analysis of data; AI, MGS, IHK, FMG and PML drafting the manuscript.

\section{Potential conflicts of interest}

HZ has served at scientific advisory boards for Roche Diagnostics, Samumed, CogRx and Wave, has given lectures in symposia sponsored by Alzecure and Biogen, and is a co-founder of Brain Biomarker Solutions in Gothenburg AB, a GU Ventures-based platform company at the University of Gothenburg. The other authors declare no conflicts of interest.

\section{References}

1. Zarrouk A, Debbabi M, Bezine M, et al. Lipid Biomarkers in Alzheimer's Disease. Curr. Alzheimer Res. 2018;15(4):303-312.

2. Gamba P, Testa G, Gargiulo S, et al. Oxidized cholesterol as the driving force behind the development of Alzheimer's disease. Front. Aging Neurosci. $2015 ; 7: 119$.

3. Hughes TM, Rosano C, Evans RW, Kuller LH. Brain Cholesterol Metabolism, Oxysterols, and Dementia. J Alzheimers Dis 2013;33(4):891-911.

4. Dias IHK, Mistry J, Fell S, et al. Oxidized LDL lipids increase $\beta$-amyloid production by SH-SY5Y cells through glutathione depletion and lipid raft 
formation. Free Radic. Biol. Med. 2014;75:48-59.

5. Wnętrzak A, Chachaj-Brekiesz A, Janikowska-Sagan M, Dynarowicz-Latka P. Influence of $7 \alpha$-hydroxycholesterol on sphingomyelin and sphingomyelin/phosphatidylcholine films - The Langmuir monolayer study complemented with theoretical calculations. Biochim. Biophys. Acta Biomembr. 2019;1861(4):861-870.

6. Phan HTT, Hata T, Morita M, et al. The effect of oxysterols on the interaction of Alzheimer's amyloid beta with model membranes. Biochim. Biophys. Acta 2013;1828:2487-2495.

7. Famer D, Meaney S, Mousavi M, et al. Regulation of $\alpha$ - and $\beta$-secretase activity by oxysterols: Cerebrosterol stimulates processing of APP via the $\alpha$-secretase pathway. Biochem. Biophys. Res. Commun. 2007;359(1):46-50.

8. Marwarha G, Raza S, Prasanthi JRP, Ghribi O. Gadd153 and NF-кB Crosstalk Regulates 27-Hydroxycholesterol-Induced Increase in BACE1 and $\beta$-Amyloid Production in Human Neuroblastoma SH-SY5Y Cells. PLoS One 2013;8(8):e70773.

9. Popp J, Meichsner S, Kölsch H, et al. Cerebral and extracerebral cholesterol metabolism and CSF markers of Alzheimer's disease. Biochem. Pharmacol. $2013 ; 86(1): 37-42$.

10. Leoni V, Solomon A, Lövgren-Sandblom A, et al. Diagnostic power of 24Shydroxycholesterol in cerebrospinal fluid: candidate marker of brain health. J. Alzheimers. Dis. 2013;36(4):739-47.

11. Shafaati M, Solomon A, Kivipelto M, et al. Levels of ApoE in cerebrospinal fluid are correlated with Tau and 24S-hydroxycholesterol in patients with cognitive disorders. Neurosci. Lett. 2007;425(2):78-82. 
12. Tournier J-D, Mori S, Leemans A, Morgan RH. Diffusion Tensor Imaging and Beyond. Magn. Reson. Med. 2011;65(6):1532-1556.

13. Alexander AL, Lee JE, Lazar M, Field AS. Diffusion Tensor Imaging of the Brain. Neurotherapeutics 2007;4(3):316-329.

14. Warstadt NM, Dennis EL, Jahanshad N, et al. Serum Cholesterol and Variant in Cholesterol-Related Gene CETP Predict White Matter Microstructure and for the Alzheimer's Disease Neuroimaging Initiative (ADNI) *. Neurobiol Aging 2014;35(11):2504-2513.

15. Ryu C-W, Coutu J-P, Greka A, et al. Differential associations between systemic markers of disease and white matter tissue health in middle-aged and older adults. J. Cereb. Blood Flow Metab. 2017;37(11):3568-3579.

16. Williams VJ, Leritz EC, Shepel J, et al. Interindividual variation in serum cholesterol is associated with regional white matter tissue integrity in older adults. Hum Brain Mapp 2013;34(8):1826-1841.

17. Kumar A, Singh S, Verma A, Mishra VN. Proteomics based identification of differential plasma proteins and changes in white matter integrity as markers in early detection of mild cognitive impaired subjects at high risk of Alzheimer's disease. Neurosci. Lett. 2018;676:71-77.

18. Li K-C, Luo X, Zeng Q-Z, et al. Distinct Patterns of Interhemispheric Connectivity in Patients With Early- and Late-Onset Alzheimer's Disease. Front. Aging Neurosci. 2018;10:261.

19. Vipin A, Ng KK, Ji F, et al. Amyloid burden accelerates white matter degradation in cognitively normal elderly individuals. Hum. Brain Mapp. 2019;

20. Al-Janabi OM, Brown CA, Bahrani AA, et al. Distinct White Matter Changes Associated with Cerebrospinal Fluid Amyloid- $\beta 1-42$ and Hypertension. J. 
Alzheimer's Dis. 2018;66(3):1095-1104.

21. Morris JC. The Clinical Dementia Rating (CDR): current version and scoring rules. Neurology 1993;43(11):2412-4.

22. Jenkinson M, Beckmann CF, Behrens TEJ, et al. FSL. Neuroimage 2012;62(2):782-790.

23. Smith SM, Jenkinson M, Woolrich MW, et al. Advances in functional and structural MR image analysis and implementation as FSL. Neuroimage 2004;23:S208-S219.

24. Woolrich MW, Jbabdi S, Patenaude B, et al. Bayesian analysis of neuroimaging data in FSL. Neuroimage 2009;45(1):S173-S186.

25. Smith SM. Fast robust automated brain extraction. Hum. Brain Mapp. 2002;17(3):143-155.

26. Andersson JLR, Jenkinson M, Smith S. Non-linear registration aka Spatial normalisation FMRIB Technical Report TR07JA2. 2007.

27. Rueckert D, Sonoda LI, Hayes C, et al. Nonrigid registration using free-form deformations: application to breast MR images. IEEE Trans. Med. Imaging 1999;18(8):712-721.

28. Smith SM, Jenkinson M, Johansen-Berg H, et al. Tract-based spatial statistics: Voxelwise analysis of multi-subject diffusion data. Neuroimage 2006;31(4):1487-1505.

29. Duits FH, Martinez-Lage P, Paquet C, et al. Performance and complications of lumbar puncture in memory clinics: Results of the multicenter lumbar puncture feasibility study. Alzheimers.Dement. 2016;12(2):154-163.

30. Lleó A, Alcolea D, Martínez-Lage P, et al. Longitudinal cerebrospinal fluid biomarker trajectories along the Alzheimer's disease continuum in the 
BIOMARKAPD study. Alzheimer's Dement. 2019;15(6):742-753.

31. Dias IHK, Milic I, Lip GYH, et al. Simvastatin reduces circulating oxysterol levels in men with hypercholesterolaemia. Redox Biol. 2018;16:139-145.

32. Blazquez L, De JD, Ruiz-Martinez J, et al. Genes related to iron metabolism and susceptibility to Alzheimer's disease in Basque population. Neurobiol.Aging 2007;28(12):1941-1943.

33. Fazekas F, Chawluk JB, Alavi A, et al. MR signal abnormalities at $1.5 \mathrm{~T}$ in Alzheimer's dementia and normal aging. AJR Am.J.Roentgenol. $1987 ; 149(2): 351-356$.

34. Smith SM, Nichols TE. Threshold-free cluster enhancement: Addressing problems of smoothing, threshold dependence and localisation in cluster inference. Neuroimage 2009;44(1):83-98.

35. Zhang R, Beyer F, Lampe L, et al. White matter microstructural variability mediates the relation between obesity and cognition in healthy adults. Neuroimage 2018;172(December 2017):239-249.

36. Wang R, Fratiglioni L, Laukka EJ, et al. Effects of vascular risk factors and APOE 4 on white matter integrity and cognitive decline. Neurology 2015;84(11):1128-1135.

37. Sato Y, Ishihara N, Nagayama D, et al. 7-ketocholesterol induces apoptosis of MC3T3-E1 cells associated with reactive oxygen species generation, endoplasmic reticulum stress and caspase-3/7 dependent pathway. Mol. Genet. Metab. Reports 2017;10:56-60.

38. Mateos L, Ismail MAM, Gil-Bea FJ, et al. Upregulation of brain renin angiotensin system by 27-hydroxycholesterol in Alzheimer's disease. J. Alzheimer's Dis. 2011;24(4):669-679. 
39. Popp J, Meichsner S, Kölsch H, et al. Cerebral and extracerebral cholesterol metabolism and CSF markers of Alzheimer's disease. Biochem. Pharmacol. $2013 ; 86(1): 37-42$.

40. Popp J, Lewczuk P, Kölsch H, et al. Cholesterol metabolism is associated with soluble amyloid precursor protein production in Alzheimer's disease. J. Neurochem. 2012;123(2):310-316.

41. Testa G, Staurenghi E, Zerbinati C, et al. Changes in brain oxysterols at different stages of Alzheimer's disease_ Their involvement in neuroinflammation. Redox Biol. 2016;10:24-33.

42. Brown AJ, Jessup W. Oxysterols: Sources, cellular storage and metabolism, and new insights into their roles in cholesterol homeostasis. Mol. Aspects Med. 2009;30(3):111-122.

43. Phan HTT, Vestergaard MC, Baek K, et al. Localization of amyloid beta (A $\beta 1$ 42) protofibrils in membrane lateral compartments: Effect of cholesterol and 7Ketocholesterol. FEBS Lett. 2014;588(18):3483-3490.

44. Kim DH, Frangos JA. Effects of Amyloid b-Peptides on the Lysis Tension of Lipid Bilayer Vesicles Containing Oxysterols. Biophys. J. 2008;95(2):620-628.

45. Phan HTT, Shimokawa N, Sharma N, et al. Strikingly different effects of cholesterol and 7-ketocholesterol on lipid bilayer-mediated aggregation of amyloid beta (1-42). Biochem. Biophys. Reports 2018;14:98-103.

46. Zhao $\mathrm{LN}$, Long $\mathrm{H}, \mathrm{Mu} \mathrm{Y}$, Chew LY. The toxicity of amyloid $\beta$ oligomers. Int. J. Mol. Sci. 2012;13(6):7303-27.

47. Khalil M, Teunissen CE, Otto M, et al. Neurofilaments as biomarkers in neurological disorders. Nat. Rev. Neurol. 2018;14(10):577-589.

48. Song S-K, Yoshino J, Le TQ, et al. Demyelination increases radial diffusivity in 
corpus callosum of mouse brain. Neuroimage 2005;26(1):132-140.

49. Li Y, Feng F, Lin P, et al. Cognition-related white matter integrity dysfunction in Alzheimer's disease with diffusion tensor image. Brain Res. Bull. 2018;143:207216.

50. Jones DK, Knösche TR, Turner R. White matter integrity, fiber count, and other fallacies: The do's and don'ts of diffusion MRI. Neuroimage 2013;73:239-254. 


\section{Figure legends}

Figure 1: Association between 7K-C and white matter microstructure. Data adjusted for age, sex and white matter hyperintensities (family-wise error-corrected, thresholded at $\mathrm{p}<0.05, \mathrm{n}=127$ ). Results are shown as an overlay on the FSL standard T1 template brain (coordinates according to MINI152 template). Abbreviations: 7K-C, 7-ketocholesterol; $\mathrm{L}$, left; R, right, FA, fractional anisotropy; $\mathrm{MD}$, mean diffusivity; $\mathrm{AxD}$, axial diffusivity; $\mathrm{RD}$, radial diffusivity.

Figure 2: Association between $A \beta_{42}$ and white matter microstructure. Note: analyses were performed in the voxels were 7-ketocholesterol showed significant effect. Adjusted for age, sex, white matter hyperintensities and $A P O E$ genotype. Abbreviations: $\mathrm{A} \beta_{42}, \beta$-amyloid $1-42 ; \mathrm{FA}$, fractional anisotropy; $\mathrm{MD}$, mean diffusivity; $\mathrm{AxD}$, axial diffusivity; RD, radial diffusivity. 


\section{Tables}

Table 1: Sample characteristics

\begin{tabular}{|c|c|c|c|}
\hline Characteristic & All sample $(\mathrm{n}=153)$ & Sample with MRI $(\mathrm{n}=127)$ & $\mathrm{p}^{\mathrm{a}}$ \\
\hline Age, years & $57.09(53.06-62.12)$ & $56.85(53.01-62.35)$ & 0.664 \\
\hline Sex ( $\%$ female $)$ & $67(43.8)$ & $78(61.4)$ & 0.005 \\
\hline Education, years & $14(11-17)$ & $14.00(11-17)$ & 0.525 \\
\hline MMSE, score & $29(28-30)$ & $29.00(28-30)$ & 0.719 \\
\hline$A P O E \& 4$ carrier & $36(23.5)$ & $27(21.3)$ & 0.202 \\
\hline Fazekas scale, n (\%) & & & 0.953 \\
\hline 0 & $86(56.6)$ & $71(55.9)$ & \\
\hline 1 & $52(34.2)$ & $44(34.6)$ & \\
\hline 2 & $9(5.9)$ & $8(6.3)$ & \\
\hline 3 & $5(3.3)$ & $4(3.1)$ & \\
\hline 24OH-C, ng/dl & $12.47(9.70-19.05)$ & $12.38(9.70-19.06)$ & 0.973 \\
\hline $27 \mathrm{OH}-\mathrm{C}, \mathrm{ng} / \mathrm{ml}$ & $2.28(1.24)$ & $2.29(1.10)$ & 0.924 \\
\hline $7 \mathrm{~K}-\mathrm{C}, \mathrm{ng} / \mathrm{dl}$ & $97.18(45.99-174.66)$ & $93.71(45.40-146.12)$ & 0.066 \\
\hline $7 \beta \mathrm{OH}-\mathrm{C}, \mathrm{ng} / \mathrm{dl}$ & $19.46(12.85-33.88)$ & $19.65(12.85-34.54)$ & 0.915 \\
\hline $\mathrm{A} \beta_{42}, \mathrm{pg} / \mathrm{ml}$ & $478.73(152.22)$ & $478.31(154.02)$ & 0.939 \\
\hline t-tau, $\mathrm{pg} / \mathrm{ml}$ & $268.40(201.87-336.76)$ & $270.87(194.24-353.65)$ & 0.451 \\
\hline p-tau, pg/ml & $38.50(30-46)$ & $39.00(30-47)$ & 0.678 \\
\hline YKL40, pg/ml & $121759.67(92656.61-153900.96)$ & $119796.16(88460.09-153787.68)$ & 0.536 \\
\hline $\mathrm{NfL}, \mathrm{pg} / \mathrm{ml}$ & $501.86(397.22-630.78)$ & $501.86(391.12-630.78)$ & 0.730 \\
\hline
\end{tabular}

Note. Significant effects are shown in bold. Mean (Standard Deviation) of measures, median (p25-p75) and in categorical variables, n (\%). 24OH-C, 24-hidroxycholesterol; 27OH-C, 27-hidroxycholesterol; 7K-C, 7 ketocholesterol; 7ßOH-C, 7 $\beta$-hidroxycholesterol; MMSE, Mini Mental Status Examination; APOE, Apolipoprotein E; NfL, Neurofilament Light

a. Comparison between participants with and without MRI. 
Table 2: Relationship between CSF oxysterols and AD CSF biomarkers

\begin{tabular}{|c|c|c|c|c|c|c|}
\hline & \multicolumn{2}{|l|}{$\mathbf{A} \boldsymbol{\beta}_{42}$} & \multicolumn{2}{|l|}{ t-tau } & \multicolumn{2}{|l|}{ p-tau } \\
\hline & $\mathrm{B}(95 \% \mathrm{IC})$ & $\mathrm{p}$ & $\mathrm{B}(95 \% \mathrm{IC})$ & $\mathrm{p}$ & $\mathrm{B}(95 \% \mathrm{IC})$ & $\mathrm{p}$ \\
\hline \multicolumn{7}{|l|}{ Model 1} \\
\hline $24 \mathrm{OH}-\mathrm{C}$ & $-0.91(-3.31-1.49)$ & 0.457 & $-0.19(-1.82-1.45)$ & 0.821 & $0.01(-0.19-0.21)$ & 0.939 \\
\hline Age & $1.35(-2.23-4.92)$ & 0.460 & $6.76(4.33-9.19)$ & 0.000 & $0.77(0.48-1.07)$ & 0.000 \\
\hline Gender: F & $-4.39(-52.61-43.84)$ & 0.858 & $-7.42(-40.26-25.42)$ & 0.658 & $0.08(-3.89-4.06)$ & 0.967 \\
\hline Gender: $\mathrm{M}$ & $0^{\mathrm{a}}$ & & $0^{\mathrm{a}}$ & & $0^{\mathrm{a}}$ & \\
\hline APOE4+ & $-52.10(-108.91-4.70)$ & 0.072 & $21.37(-17.14-59.88)$ & 0.277 & $2.58(-2.07-7.23)$ & 0.277 \\
\hline APOE4- & $0^{\mathrm{a}}$ & & $0^{\mathrm{a}}$ & & $0^{\mathrm{a}}$ & \\
\hline \multicolumn{7}{|l|}{ Model 2} \\
\hline $27 \mathrm{OH}-\mathrm{C}$ & $5.86(-13.85-25.57)$ & 0.560 & $1.98(-11.43-15.39)$ & 0.772 & $0.91(-0.70-2.53)$ & 0.266 \\
\hline Age & $1.31(-2.29-4.90)$ & 0.476 & $6.78(4.34-9.22)$ & 0.000 & $0.80(0.50-1.09)$ & 0.000 \\
\hline Gender: F & $-5.68(-54.74-43.38)$ & 0.820 & $-6.52(-39.89-26.84)$ & 0.702 & $0.06(-3.96-4.09)$ & 0.975 \\
\hline Gender: $\mathrm{M}$ & $0^{\mathrm{a}}$ & & $0^{\mathrm{a}}$ & & $0^{\mathrm{a}}$ & \\
\hline APOE4+ & $-54.15(-112.04-3.74)$ & 0.067 & $23.42(-15.78-62.63)$ & 0.242 & $2.82(-1.89-7.54)$ & 0.241 \\
\hline APOE4- & $0^{\mathrm{a}}$ & & $0^{\mathrm{a}}$ & & $0^{\mathrm{a}}$ & \\
\hline \multicolumn{7}{|l|}{ Model 3} \\
\hline $7 \mathrm{~K}-\mathrm{C}$ & $-0.20(-0.39--0.01)$ & 0.041 & $-0.06(-0.19-0.07)$ & 0.338 & $-0.01(-0.03-0.00)$ & 0.170 \\
\hline Age & $1.44(-2.08-4.96)$ & 0.422 & $6.79(4.38-9.20)$ & 0.000 & $0.79(0.50-1.08)$ & 0.000 \\
\hline Gender: F & $-3.17(-51.10-44.76)$ & 0.897 & $-6.33(-39.23-26.57)$ & 0.706 & $0.17(-3.80-4.14)$ & 0.934 \\
\hline Gender: $\mathrm{M}$ & $0^{\mathrm{a}}$ & & $0^{\mathrm{a}}$ & & $0^{\mathrm{a}}$ & \\
\hline APOE4+ & $-54.87(-111.25-1.51)$ & 0.056 & $19.96(-18.58-58.51)$ & 0.310 & $2.42(-2.23-7.06)$ & 0.308 \\
\hline APOE4- & $0^{\mathrm{a}}$ & & $0^{\mathrm{a}}$ & & $0^{\mathrm{a}}$ & \\
\hline \multicolumn{7}{|l|}{ Model 4} \\
\hline $7 \beta-C$ & $0.44(-0.43-1.32)$ & 0.321 & $-0.42(-1.00-0.16)$ & 0.160 & $-0.05(-0.12-0.02)$ & 0.201 \\
\hline Age & $0.91(-2.68-4.49)$ & 0.619 & $6.86(4.44-9.29)$ & 0.000 & $0.79(0.49-1.08)$ & 0.000 \\
\hline Gender: F & $-6.39(-55.54-42.76)$ & 0.799 & $-9.26(-42.55-24.04)$ & 0.586 & $-0.18(-4.21-3.85)$ & 0.930 \\
\hline Gender: $\mathrm{M}$ & $0^{\mathrm{a}}$ & & $0^{\mathrm{a}}$ & & $0^{\mathrm{a}}$ & \\
\hline APOE4+ & $-55.91(-113.10-1.29)$ & 0.055 & $23.15(-15.44-61.75)$ & 0.240 & $2.75(-1.91-7.41)$ & 0.248 \\
\hline APOE4- & $0^{\mathrm{a}}$ & & $0^{\mathrm{a}}$ & & $0^{\mathrm{a}}$ & \\
\hline
\end{tabular}

Note. Significant effects are shown in bold. B, beta coefficient; CI, confidence interval; APOE4+ = apolipoprotein E $\varepsilon 4$ carrier, APOE4- = apolipoprotein E $\varepsilon 4$ non-carrier; 24OH-C, 24-hidroxycholesterol; 27OH-C, 27 -

hidroxycholesterol; 7K-C, 7-ketocholesterol; 7 $\beta \mathrm{OH}-\mathrm{C}, 7 \beta$-hydroxycholesterol; $\mathrm{A} \beta_{42}=\beta$-amyloid - $_{-42}$; t-tau $=$ total-tau; p-tau $=$ phosphorylated tau

a. Set to zero because this parameter is redundant. 
Table 3. Distribution and proportion of significant voxels for the association between 7K-C and cognitive regions DTI indexes.

\begin{tabular}{llcccc}
\hline & Region (N ${ }^{\circ}$ of voxels) $)^{\mathrm{a}}$ & \multicolumn{3}{c}{${\text { Number of significant voxels }(\%)^{\mathrm{b}}}$} & RD \\
\cline { 3 - 6 } & & FA & MD & AxD & $2(0.02)$ \\
\hline CC & Genu (8851) & $429(4.85)$ & $470(5.31)$ & $98(1.11)$ & $21(0.15)$ \\
& Body (1371) & $247(1.80)$ & $658(4.80)$ & 0 & $387(3.04)$ \\
& Splenium (12729) & $223(1.75)$ & $524(4.12)$ & $116(0.91)$ & $47(7.13)$ \\
Fornix & Column+body (659) & 0 & $50(7.59)$ & $53(8.04)$ & $42(3.74)$ \\
& Cres/stria terminalis Right (1124) & 0 & $36(3.20)$ & $16(1.42)$ & $61(5.42)$ \\
& Cres/stria terminalis Left (1125) & 0 & $68(6.04)$ & $55(4.89)$ & $255(11.45)$ \\
ILF & Right (2228) & 0 & $298(13.38)$ & $231(10.37)$ & $35(1.57)$ \\
& Left (2231) & 0 & $258(11.56)$ & $240(10.76)$ & $617(9.34)$ \\
SLF & Right (6607) & $118(1.79)$ & $578(8.75)$ & $332(5.02)$ & $397(6.01)$ \\
& Left (6605) & 0 & $447(6.77)$ & $4(0.06)$ & 0 \\
GC & Right (2342) & 0 & $31(1.32)$ & 0 & 0 \\
& Left (2751) & $16(0.58)$ & $42(1.53)$ & 0 & $247(19.98)$ \\
Hippoc. & Right (1236) & $239(19.34)$ & $229(18.53)$ & $28(2.27)$ & 0 \\
& Left (1155) & 0 & $120(10.39)$ & 0 & 0 \\
& Right (380) & 0 & $38(10.00)$ & 0 & 0 \\
& Left (376) & 0 & 0 & 0 & 0
\end{tabular}

Note. Number of significant voxels (FEW-corrected and $\mathrm{p}<0.05$ ). CC, corpus callosum; ILF, inferior longitudinal fasciculus; SLF, superior longitudinal fasciculus; GC, gyrus cingulum; Hippoc., hippocampus; UF, uncinated fasciculus

a. In parenthesis, the number of voxels according to ICBM-DTI-81 white matter labels atlas

b. In parenthesis the proportion of significant voxels with respect to the total number of voxels in this area 
Table 4: Effect of 7K-C and cognitive regions WM microstructure on $\mathrm{A} \beta_{42}$ levels.

\begin{tabular}{|c|c|c|c|c|c|c|}
\hline & \multicolumn{2}{|l|}{ FRNX (MD) } & \multicolumn{2}{|l|}{ FRNX (AxD) } & \multicolumn{2}{|l|}{ FRNX (RD) } \\
\hline & $\mathrm{B}(95 \% \mathrm{IC})$ & $\mathrm{p}^{\mathrm{a}}$ & $\mathrm{B}(95 \% \mathrm{IC})$ & $\mathrm{p}^{\mathrm{a}}$ & $\mathrm{B}(95 \% \mathrm{IC})$ & $\mathrm{p}^{\mathrm{a}}$ \\
\hline $7 \mathrm{~K}-\mathrm{C}$ & $-0.15(-0.76-0.46)$ & 0.635 & $-0.13(-0.98-0.72)$ & 0.771 & $-0.15(-0.65-0.36)$ & 0.575 \\
\hline MD & $-121.19(-207.72-34.66)$ & 0.006 & & & & \\
\hline $7 \mathrm{~K}-\mathrm{C} * \mathrm{MD}$ & $0.02(-0.29-0.34)$ & 0.890 & & & & \\
\hline $\mathrm{AxD}$ & & & $-131.74(-230.42-33.06)$ & 0.009 & & \\
\hline $7 \mathrm{~K}-\mathrm{C} * \mathrm{AxD}$ & & & $0.01(-0.34-0.36)$ & 0.955 & & \\
\hline $\mathrm{RD}$ & & & & & $-112.62(-192.35-32.89)$ & 0.006 \\
\hline \multirow[t]{3}{*}{$7 \mathrm{~K}-\mathrm{C} * \mathrm{RD}$} & & & & & $0.02(-0.28-0.32)$ & 0.884 \\
\hline & \multicolumn{2}{|l|}{ CC-S (FA) } & \multicolumn{2}{|l|}{ CC-S (AxD) } & \multicolumn{2}{|l|}{ CC-B (MD) } \\
\hline & $\mathrm{B}(95 \% \mathrm{IC})$ & $\mathrm{p}^{\mathrm{a}}$ & B (95\%IC) & $\mathrm{p}^{\mathrm{a}}$ & B (95\%IC) & $\mathrm{p}^{\mathrm{a}}$ \\
\hline $7 \mathrm{~K}-\mathrm{C}$ & $0.65(-4.96-6.26)$ & 0.820 & $-2.50(-6.14-1.13)$ & 0.177 & $0.80(-2.61-4.21)$ & 0.645 \\
\hline FA & $1.09(-0.39-2.57)$ & 0.148 & & & & \\
\hline $7 \mathrm{~K}-\mathrm{C} * \mathrm{FA}$ & $-0.001(-0.008-0.006)$ & 0.796 & & & & \\
\hline $\mathrm{AxD}$ & & & $-472.22(-900.19-44.26)$ & 0.031 & & \\
\hline $7 \mathrm{~K}-\mathrm{C}^{*} \mathrm{AxD}$ & & & $1.54(-0.80-3.89)$ & 0.198 & & \\
\hline MD & & & & & $-457.53(-1498.56-583.51)$ & 0.389 \\
\hline \multirow[t]{3}{*}{$7 \mathrm{~K}-\mathrm{C} * \mathrm{MD}$} & & & & & $-1.198(-5.651-3.254)$ & 0.598 \\
\hline & \multicolumn{2}{|l|}{ CC-G (AxD) } & \multicolumn{2}{|l|}{ ILF L (AxD) } & \multicolumn{2}{|l|}{ Hippoc. R (AxD) } \\
\hline & B (95\%IC) & $\mathrm{p}^{\mathrm{a}}$ & $\mathrm{B}(95 \% \mathrm{IC})$ & $\mathrm{p}^{\mathrm{a}}$ & B (95\%IC) & $\mathrm{p}^{\mathrm{a}}$ \\
\hline $7 \mathrm{~K}-\mathrm{C}$ & $-0.31(-4.16-3.54)$ & 0.873 & $-5.42(-10.49-0.35)$ & 0.036 & $-0.02(-1.17-1.13)$ & 0.976 \\
\hline $\mathrm{AD}$ & $-440.17(-813.01-67.33)$ & 0.021 & $-722.00(-1287.77-156.23)$ & 0.012 & $248.42(57.91-438.94)$ & 0.011 \\
\hline $7 \mathrm{~K}-\mathrm{C}^{*} \mathrm{AxD}$ & $0.13(-2.30-2.56)$ & 0.914 & $3.87(0.17-7.58)$ & 0.040 & $-0.23(-1.35-0.89)$ & 0.686 \\
\hline
\end{tabular}

Note. Significant effects are shown in bold. B, beta coefficient; CI, confidence interval; $\mathrm{A} \beta_{42}=. \beta$-amyloid ${ }_{1-42}$; CC-S, corpus callosum-splenium; CC-B, corpus callosum-body; CC-G, corpus-callosum-genu; ILF L, inferior longitudinal fasciculus left; FRNX, fornix (column+body); Hippoc. R, hippocampus right, FA, fractional anisotropy; MD, mean diffusivity; AxD, axial diffusivity; RD, radial diffusivity.

a. Generalized linear models adjusted for gender, age, white matter hyperintensities and $A P O E$ genotype 
Table 5: Effect of $7 \mathrm{~K}-\mathrm{C}, \mathrm{A} \beta_{42}$ and cognitive regions axial diffusivity on NfL levels.

\begin{tabular}{|c|c|c|c|c|}
\hline & \multicolumn{2}{|l|}{ FRNX } & \multicolumn{2}{|l|}{ CC-S } \\
\hline & $\mathrm{B}(95 \% \mathrm{IC})$ & $\mathrm{p}^{\mathrm{a}}$ & $\mathrm{B}(95 \% \mathrm{IC})$ & $\mathrm{p}^{\mathrm{a}}$ \\
\hline \multicolumn{5}{|l|}{ Model 1} \\
\hline $\mathrm{AxD}$ & $-3.37(-4.88--1.86)$ & 0.000 & $-633.11(-1425.09-158.87)$ & 0.117 \\
\hline $7 \mathrm{~K}-\mathrm{C}$ & $-202.83(-377.69--27.97)$ & 0.023 & $-8.19(-14.91--1.46)$ & 0.017 \\
\hline $\mathrm{AxD} * 7 \mathrm{~K}-\mathrm{C}$ & $1.54(0.92-2.15)$ & 0.000 & $5.52(1.17-9.86)$ & 0.013 \\
\hline \multicolumn{5}{|l|}{ Model 2} \\
\hline $\mathrm{AxD}$ & $331.43(-121.02-783.88)$ & 0.151 & $-456.89(-2281.61-1367.83)$ & 0.624 \\
\hline $\mathrm{A} \beta_{42}$ & $1.17(-0.84-3.18)$ & 0.254 & $-2.13(-7.69-3.43)$ & 0.452 \\
\hline \multirow[t]{3}{*}{$\mathrm{AxD} * \mathrm{~A} \beta_{1-42}$} & $-0.42(-1.36-0.53)$ & 0.388 & $1.60(-2.16-5.37)$ & 0.404 \\
\hline & \multicolumn{2}{|l|}{ CC-G } & \multicolumn{2}{|l|}{ ILF L } \\
\hline & $\mathrm{B}(95 \% \mathrm{IC})$ & $\mathrm{p}^{\mathrm{a}}$ & $\mathrm{B}(95 \% \mathrm{IC})$ & $\mathrm{p}^{\mathrm{a}}$ \\
\hline \multicolumn{5}{|l|}{ Model 1} \\
\hline $\mathrm{AxD}$ & $-855.54(-1523.47--187.60)$ & 0.012 & $-447.54(-1515.27-620.18)$ & 0.411 \\
\hline $7 \mathrm{~K}-\mathrm{C}$ & $-14.87(-21.77--7.97)$ & 0.000 & $2.84(-6.72-12.41)$ & 0.561 \\
\hline $\mathrm{AxD} * 7 \mathrm{~K}-\mathrm{C}$ & $9.61(5.26-13.96)$ & 0.000 & $-1.75(-8.73-5.24)$ & 0.624 \\
\hline \multicolumn{5}{|l|}{ Model 2} \\
\hline AxD & $-216.69(-1753.82-1320.44)$ & 0.782 & $1016.47(-1347.23-3380.17)$ & 0.399 \\
\hline $\mathrm{A} \beta_{42}$ & $-1.28(-5.60-3.05)$ & 0.563 & $3.79(-2.51-10.10)$ & 0.238 \\
\hline$A x D * A \beta_{42}$ & $1.01(-1.85-3.86)$ & 0.490 & $-2.75(-7.55-2.05)$ & 0.261 \\
\hline
\end{tabular}

Hippoc. $\mathbf{R}$

\begin{tabular}{lcc} 
& $\mathrm{B}(95 \% \mathrm{IC})$ & $\mathrm{p}^{\mathrm{a}}$ \\
\cline { 2 - 3 } Model 1 & \\
\hline AxD & $-45.98(-413.78-321.81)$ & 0.806 \\
$7 \mathrm{~K}-\mathrm{C}$ & $0.69(-1.54-2.91)$ & 0.546 \\
$\mathrm{AxD} * 7 \mathrm{~K}-\mathrm{C}$ & $-0.30(-2.46-1.86)$ & 0.785 \\
\hline Model 2 & & \\
\hline AxD & $-19.19(-962.30-923.92)$ & 0.968 \\
$\mathrm{~A} \beta_{42}$ & $0.27(-1.42-1.97)$ & 0.750 \\
$\mathrm{AxD} * \mathrm{~A} \beta_{42}$ & $-0.07(-1.89-1.76)$ & 0.942
\end{tabular}

Note. Significant effects are shown in bold. B, beta coefficient; $\mathrm{CI}$, confidence interval; $\mathrm{A} \beta_{42}=\beta$ amyloid $_{1-42}$; CC-S, corpus callosum-splenium; CC-G, corpus-callosum-genu; ILF L, inferior longitudinal fasciculus left; FRNX, fornix (column+body); Hippoc. R, hippocampus right, AxD, axial diffusivity; NfL, neurofilament light; 7K-C, 7-ketocholesterol.

b. Generalized linear models adjusted for gender, age, white matter hyperintensities and $A P O E$ genotype 